\title{
Visual Field Recovery - Case Report of a Patient with Left Homonymous Hemianopsia Post-Stroke
}

\author{
Marsha Davis Benshir* \\ Center for Vision Development, USA \\ *Corresponding author: Marsha Davis Benshir, Center for Vision Development, 164 W Main St Ste. B New Market, MD 21774, USA

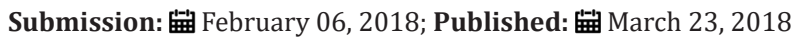

\begin{abstract}
Visual field loss from stroke occurs in up to $50 \%$ of patients requiring hospitalization for rehabilitation after stroke [1]. Of these patients many will have spontaneous recovery and others will adapt through physical and occupational therapy. Vision rehabilitation modeled on other widely used types of therapy post-stroke has been demonstrated to restore peripheral vision in some patients beyond the expected window for spontaneous recovery of peripheral vision.
\end{abstract}

\section{Introduction}

Optometrists specializing in Neuro-optometric rehabilitation have been working with physiatrists, neurologists and other rehabilitation specialist for decades, and have demonstrated that visual field recovery is possible for many patients beyond the expected time for recovery. The research proving that therapy can be effective is being done by neurologists who have demonstrated re-wiring of neural pathways with improved performance after repetitive visual-motor tasks and recovery of somatosensory and motor awareness after virtual reality (visual) stimulation [2].

In one UK study of 915 stroke patients $52 \%$ had visual field loss and $10 \%$ had no symptoms. Of the patients with visual field loss half had difficulty reading, blurry vision, diplopia, ocular motor and perceptual problems while half complained only of their peripheral vision loss. At follow up 15 patients (7.5\%) had full recovery, 78 (39\%) had improvement, and 104 (52\%) had no recovery. Two patients $(1 \%)$ had further decline of visual field. Patients with visual field loss had lower quality of life scores than stroke patients without visual impairment and patients with homonymous hemianopsia had worse overall prognosis for recovery [3].

Despite proof of neuro-plasticity the prevailing standard of care maintains that visual field recovery beyond that occurring spontaneously is not possible [4]. Patients are encouraged to learn compensatory strategies at best and to make lifestyle adjustments to avoid visually challenging tasks.

Visual field loss is estimated at 1:20 community dwelling elderly people and is associated with many functional impairments including difficulty reading, watching TV and increased risk of falling. In patients over 85 the incidence is closer to $1: 6$. In one study in Rotterdam, Netherlands, glaucoma was the leading cause of visual field loss followed by other optic nerve disease and stroke [5].

\section{Case Report}

This patient was a previously healthy 60 y.o. right-handed male who developed sudden left side facial weakness, confusion and disorientation on the way home from work. When he arrived home he had a left facial droop and headache, and his wife called EMS. According to those records his BP was 180/110. He was transported to the ED and admitted although his CT was unremarkable. MRI was performed the next morning and showed a right parieto temporal intraparenchymal hemorrhage. He decompensates and was incubated. He was transferred to another hospital for a right decompressive craniectomy with clot evacuation and ICP monitor placement. He developed aspiration pneumonia. After being medically stabilized he was transferred for acute in-patient rehabilitation. At rehabilitation he was alert and oriented x3. He was able to follow one-step commands and 2-step commands with verbal cues. He had left inattention and suspected receptive aphasia. He had PEG placed.

A vision evaluation found left homonymous hemianopsia in addition to his left hemiplegia. Pupil reflexes were present but unequal, with the left pupil larger than the right. There were no ocular motor restrictions. He did not have convergence reflex and manifested alternating exotropia at near without diplopia. His wife was present and added that he had a history of retinal detachment OD with sclera buckle. 
At discharge this patient was started on a program of vision stimulation to recover peripheral vision and binocular vision. This was practiced at home with instructions provided. He presented 10 months later with visual fields showing left homonymous hemianopsia, with partial recovery. He was working part-time and concerned about lack of continued progress in his recovery. He elected to participate in a program of intensive visual field stimulation with the goal of being able to return to driving.

This patient participated in 5 hours of in-office vision rehabilitation in addition to procedures practiced daily at home, supervised by his wife. The threshold field testing presented below demonstrates significant vision recovery of the left field with both eyes

Procedures used to stimulate peripheral vision included moving targets over a large field of view at far and near, binocular stimulation, visual motor tasks, and procedures specific to right brain function. This patient showed rapid improvements in a very short time. This is believed to be due to his relatively young age at onset of stroke and exceptionally good health and level of activity prior to stroke. He was highly motivated and had good family support. He has returned to driving and all pre-morbid activities, although he is frustrated by his inability to regain his earlier baseline function (Figure 1-4).

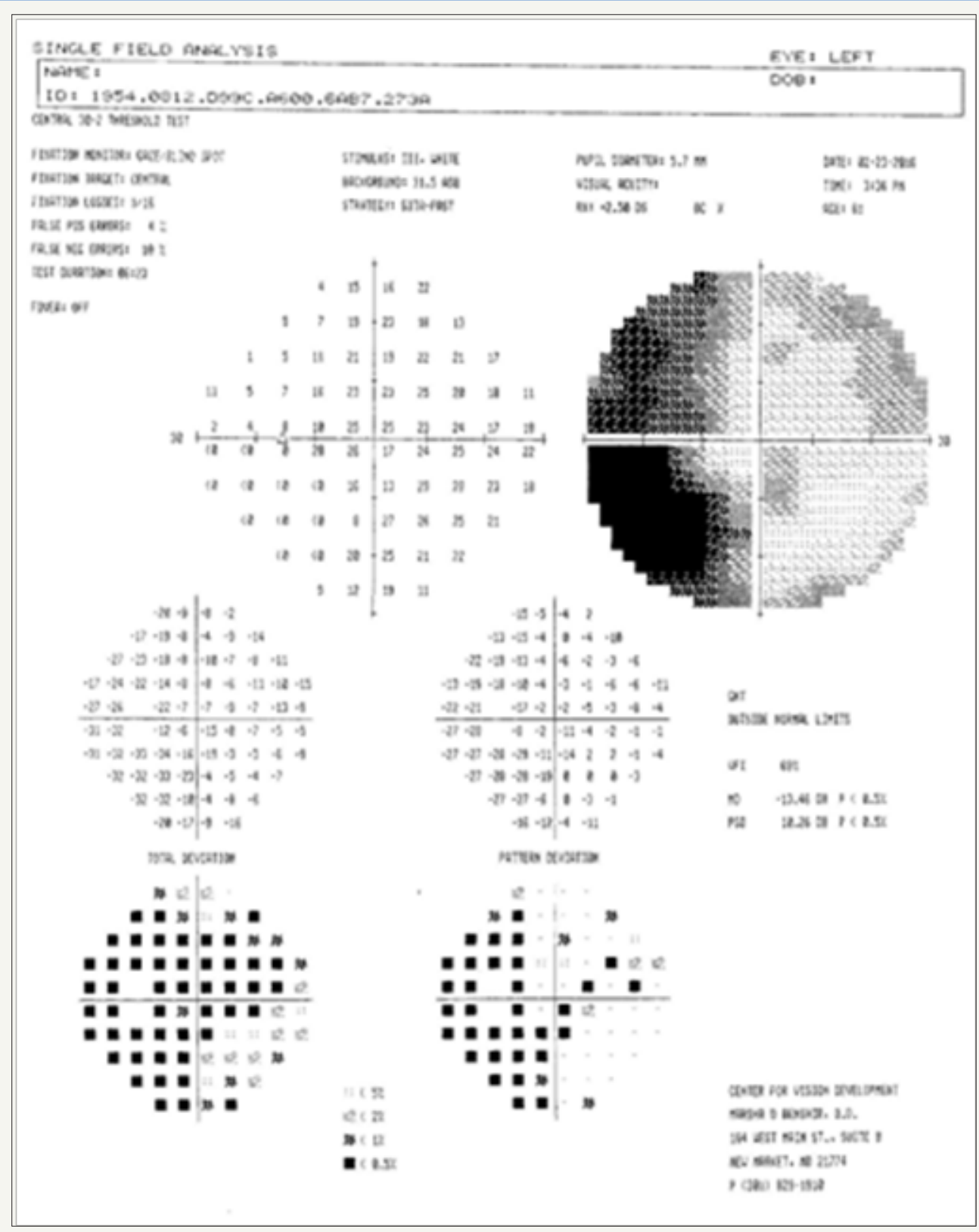

Figure 1: Right eye pre-therapy 


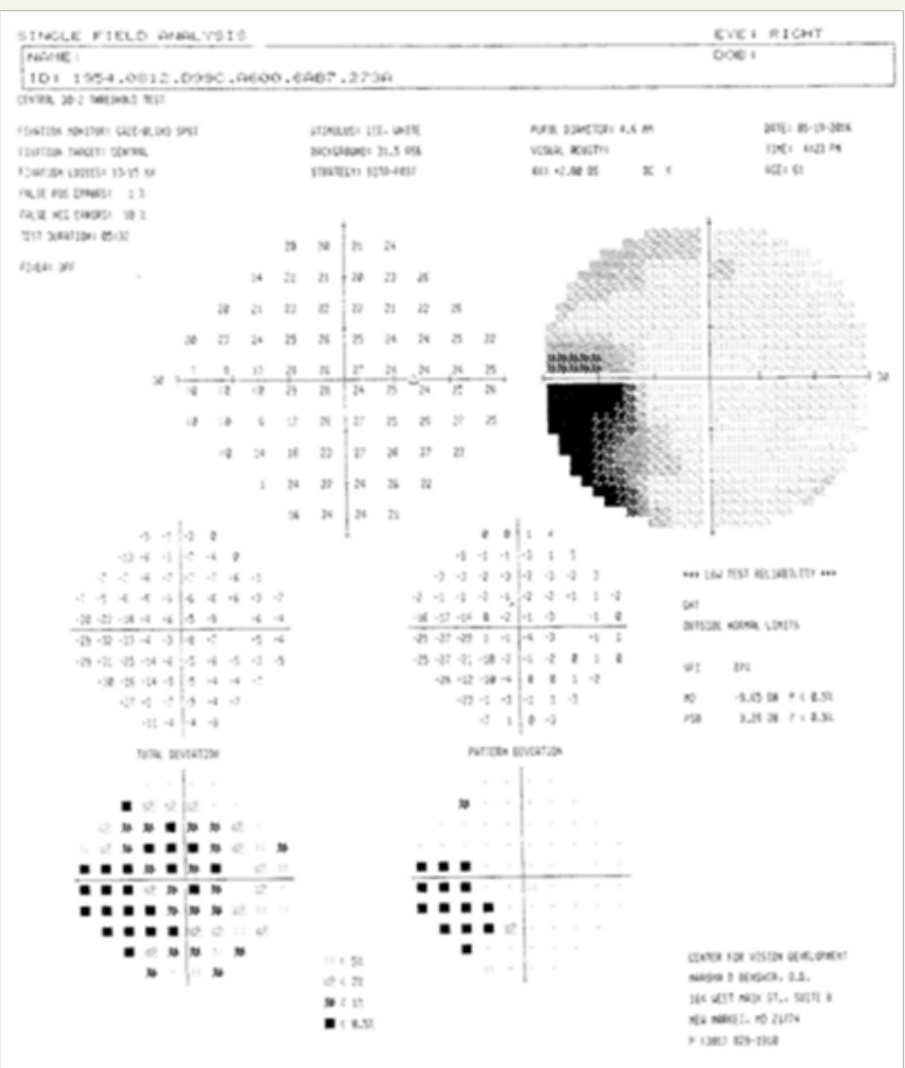

Figure 2: Right eye post-therapy

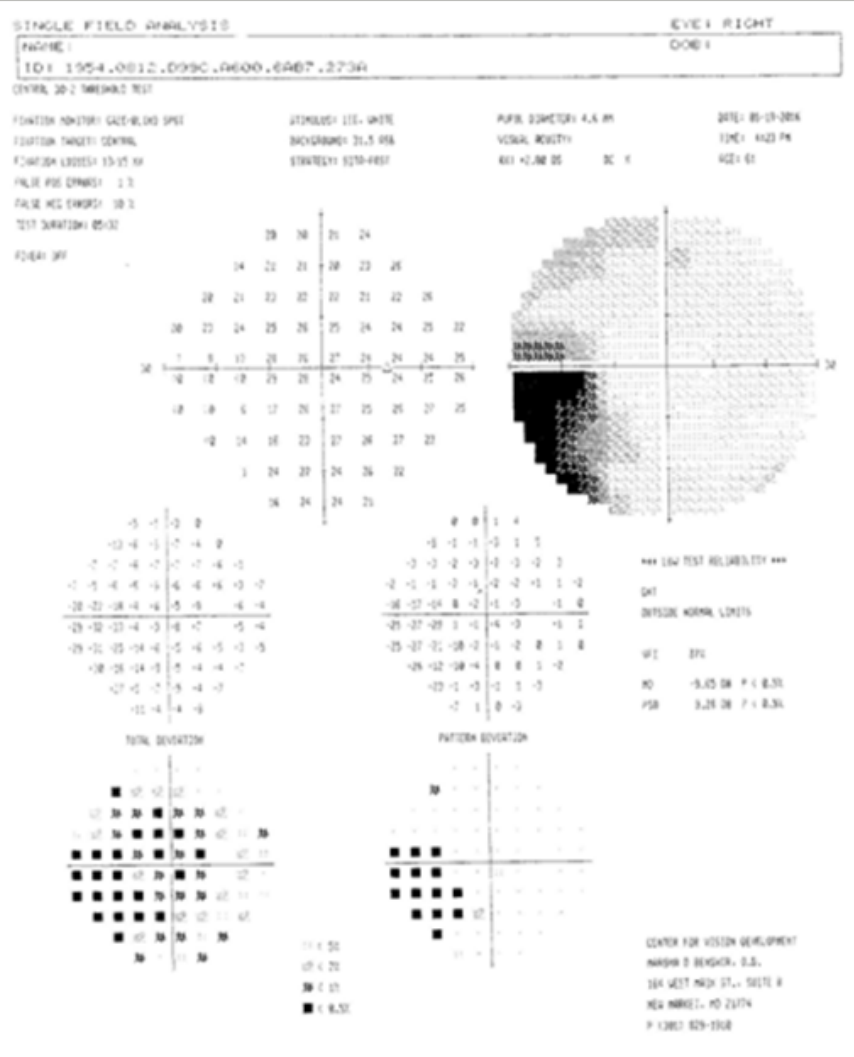

Figure 3: Left eye pre-therapy 


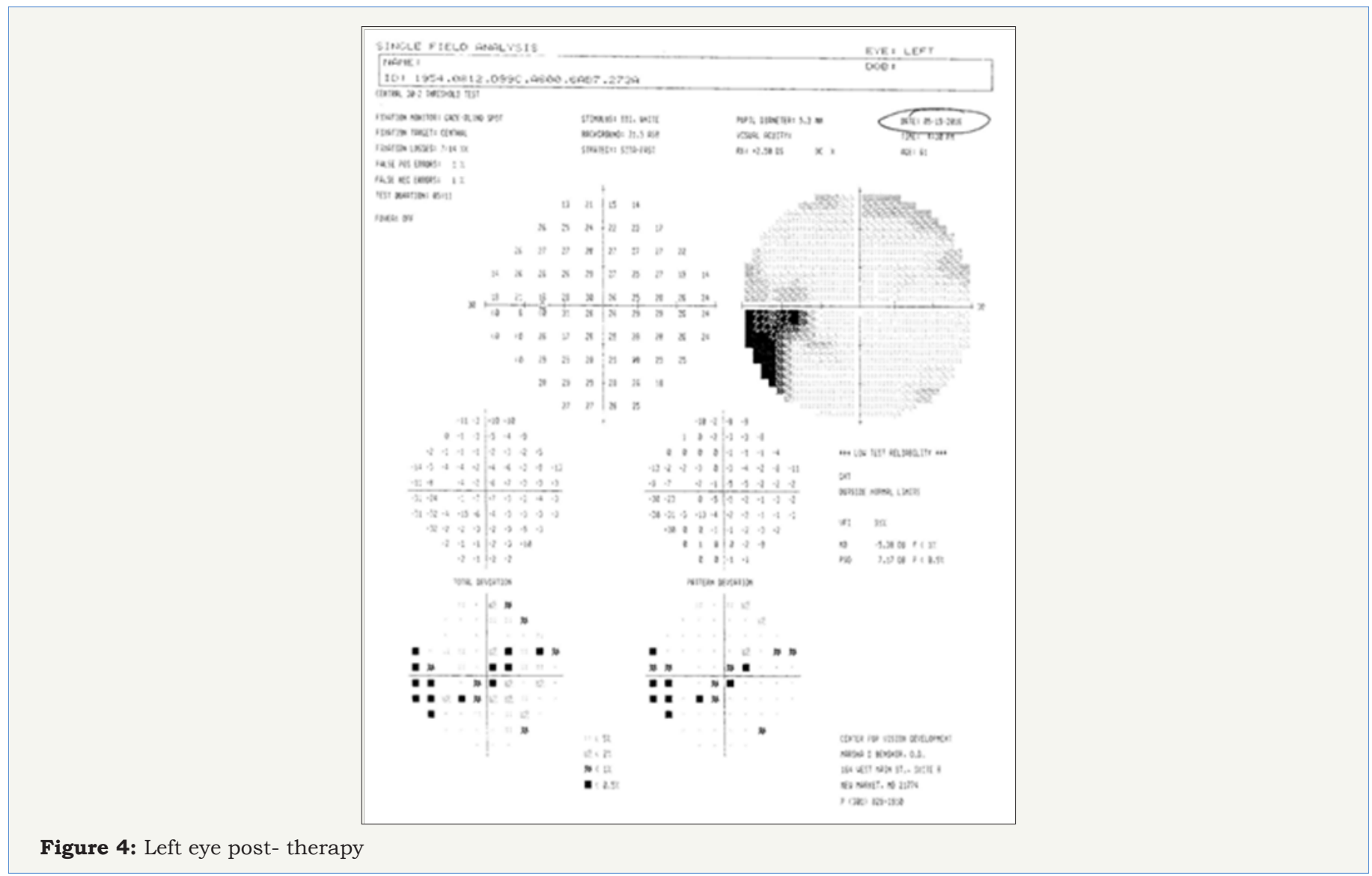

\section{Conclusion}

Neuro-optometric rehabilitation has demonstrated that visual field recovery is possible for patients with healthy eyes. Anecdotal reports are strong but research is needed, including patient reports demonstrating recovery for significant numbers of patients, to demonstrate the efficacy of therapeutic intervention.

\section{References}

1. Benshir Marsha (2016) Prevalence of Vision Disorders After Stroke: A Pilot Study to Identify the Visual Needs of Stroke Patients. Optometry and Visual Performance 4(4): 129-132.
2. Berry Anne S, Zanto TP, Clapp WC, Hardy JL, Delahunt PB, et al (2010) The influence of perceptual training on working memory in older adults. Public Library of Science one 5(7): e11537.

3. Fiona J Rowe (2013) A Prospective Profile of Visual Field Loss following Stroke: Prevalence, Type, Rehabilitation, and Outcome. Biomed Res Int. 2013: 719096.

4. ALM Pambakian, C Kennard (1997) Can visual function be restored in patients with homonymous hemianopia? Br J Ophthalmol 81(4): 324328.

5. Ramrattan RS (2001) Prevalence and causes of visual field loss in the elderly and associations with impairment in daily functioning: the Rotterdam Study. Arch Ophthalmol 119(12): 1788-1794. (c) (i) Creative Commons Attribution 4.0

For possible submissions Click Here

\section{Submit Article}

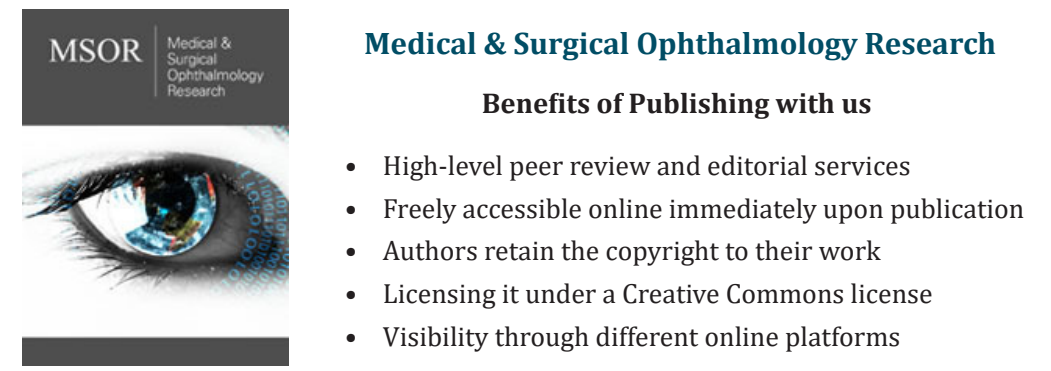

